\title{
Campylobacter jejuni Mediated Disruption of Polarized Epithelial Monolayers is Cell-Type Specific, Time Dependent, and Correlates With Bacterial Invasion
}

\author{
EYTAN WINE, VOON L. CHAN, AND PHILIP M. SHERMAN

\begin{abstract}
Department of Paediatrics [E.W., P.M.S.], University of Toronto, Toronto, Ontario, M5G 1X8, Canada; Department of Medical Genetics and Microbiology [V.L.C.], University of Toronto, Toronto, Ontario, M5S 1A8, Canada; Department of Laboratory Medicine and Pathobiology [P.M.S.], University of Toronto, Toronto, Ontario, M5G 1L5, Canada
\end{abstract}

\begin{abstract}
The precise mechanism by which the most common cause of bacterial enterocolitis in humans, Campylobacter jejuni, perturbs the intestinal mucosa remains elusive. To define effects of $C$. jejuni infection on mucosal permeability, Madin-Darby canine kidney (MDCK)-I and T84 cell monolayers were infected with $C$. jejuni for up to $48 \mathrm{~h}$. All three tested $C$. jejuni strains caused a $73-78 \%$ reduction in transepithelial electrical resistance (TER) in intestinal (T84) cell monolayers, whereas only one strain slightly reduced TER of MDCK-I cells by $25 \%$ after $48 \mathrm{~h}$ infection. Infection with C. jejuni strains also caused a 2.3-4.5-fold increase in dextran permeability, but only in T84 cells. C. jejuni infection of monolayers also caused morphologic changes in desmosomes, observed by transmission electron microscopy. The cell-type specificity, demonstrated by increased T84 monolayer permeability, correlated with higher bacterial invasion into these cells, relative to MDCK-I cells. In T84 cells, invasion and bacterial translocation preceded barrier disruption and inhibition of $C$. jejuni invasion using a pharmacological inhibitor of phosphoinositide 3-kinase, reduced the drop in TER. These findings suggest that $C$. jejuni disruption of monolayers is mediated by invasion, provide new insights into $C$. jejuni-host epithelial barrier interactions, and offer potential mechanisms of intestinal injury and chronic immune stimulation. (Pediatr Res 64: 599-604, 2008)
\end{abstract}

$\mathrm{C}$ ampylobacter jejuni is the leading cause of bacterialinduced enterocolitis across the globe $(1,2)$. The burden of disease is considerable, especially among children, both in developing countries, where infection can result in lifethreatening diarrhea, and in children from industrialized countries, who may be exposed to $C$. jejuni through travel, contaminated food, or contact with pets (3). Intestinal symptoms following infection with $C$. jejuni species are often selflimited, ranging from watery diarrhea to dysentery, although C. jejuni has also been implicated in chronic intestinal disorders, including postinfectious irritable bowel syndrome (4) and chronic inflammatory bowel diseases (IBD) $(2,5,6)$. How-

Received April 16, 2008; accepted July 12, 2008.

Correspondence: Philip M. Sherman, MD, FRCPC, Hospital for Sick Children, Room 8409, 555 University Avenue, Toronto, Ontario, Canada M5G 1X8; e-mail: philip.sherman@sickkids.ca

Supported by operating grants from the Canadian Institutes of Health Research (CIHR) and the Crohn's and Colitis Foundation of Canada (Fay Shapiro Cutler Grant in Aid of Research).

Supported by a Fellowship Award from the CIHR/Canadian Association of Gastroenterology/AstraZeneca Canada and by a University of Toronto Faculty of Medicine Postgraduate Award (E.W.).

PMS is the recipient of a Canada Research Chair in Gastrointestinal Disease. ever, microaerophilic growth requirements and the paucity of suitable animal models limit current understanding of disease pathogenesis (7).

Several factors contributing to bacterial virulence have been identified to date, including adhesion, invasion, and the cytolethal-distending-toxin. Invasion is dependent on host microtubules (8), cytolethal-distending-toxin secretion (9), and the pVir type IV secretion system homologue (10). After invasion, $C$. jejuni manipulates host signaling to avoid lysosomal degradation (11). A recently described $C$. jejuni isolate, which causes diarrhea in humans and in a ferret model of infection, despite very low invasion rates in vitro, suggests involvement of other pathogenic mechanisms (12).

The intestinal barrier is comprised of a single layer of polarized epithelial cells serving to separate the luminal content, including microbes, from the underlying mucosa. The main physical component contributing to the barrier is the apical junctional complex, which includes highly regulated tight junctions and adherens junctions, bridging between epithelial cells and connecting the cytoskeleton (13). Breaches in the epithelial barrier integrity result in penetration of luminal antigens and microbes, which stimulate pro-inflammatory responses, leading to chronic intestinal and systemic diseases, including IBD (13). Pathogenic bacteria can disrupt the mucosal barrier by secretion of toxins, transfer of effector proteins into host cells, invasion of epithelial cells, translocation across the epithelial monolayer, or by disruption of intercellular junctions (14).

The aim of this study was to characterize the effects of three prototype $C$. jejuni strains on permeability by employing polarized epithelial monolayers with different characteristics. We demonstrate herein that $C$. jejuni infection causes a time-dependent increase in permeability, which correlates with the ability of $C$. jejuni to invade and disrupt epithelial cell monolayers.

\section{METHODS}

Epithelial cells in tissue culture. HEp-2 cells were cultured in Minimal Essential Medium (MEM), 15\% heat-inactivated FCS, $0.1 \%$ sodium bicar-

\footnotetext{
Abbreviations: EHEC, enterohemorrhagic Escherichia coli; IBD, inflammatory bowel diseases; MDCK, Madin-Darby canine kidney; MOI, multiplicity of infection; PI3-K, phosphoinositide 3-kinase; TER, transepithelial electrical resistance
} 
bonate, and $2 \%$ penicillin-streptomycin. Intestine 407 were cultivated in MEM, $10 \%$ FCS, and 2\% penicillin-streptomycin. In contrast to HEp-2 and Intestine 407 cells, T84 and Madin-Darby canine kidney (MDCK)-I cells are polarized epithelial cells that form apical junctional complexes, resulting in high electrical resistance, useful for studying effects of bacteria on permeability $(15,16)$. T84 human colon cancer epithelial cells were cultured in DMEM/F-12, 10\% FCS, $2 \%$ penicillin-streptomycin, $2 \%$ sodium bicarbonate, and $0.6 \%$ L-glutamine. MDCK-I cells were grown in DMEM, $10 \%$ FCS, and $2 \%$ penicillin-streptomycin (All from GIBCO, Grand Island, NY). Cells were maintained in $25 \mathrm{~cm}^{2}$-flasks (Corning Glass Works, Corning, NY) and then grown on six-well plates or 12-well Transwells ( $6.5 \mathrm{~mm}$ diameter; 0.4 or $3 \mu$ pore size; $37^{\circ} \mathrm{C} ; 5 \% \mathrm{CO}_{2}$; Corning).

Pharmacological inhibitors. To disrupt host microtubules, monolayers were pretreated for $1 \mathrm{~h}$ before infection with $5-50 \mu \mathrm{g} / \mathrm{mL}$ colchicine (Sigma Chemical Co., Saint Louis, MO). In experiments involving a phosphoinositide 3-kinase (PI3-K) inhibitor, monolayers were pretreated with $25-100 \mu \mathrm{M}$ of either LY294002 or an inactive analog, LY303511 (Calbiochem, La Jolla, $\mathrm{CA}$ ) for $1 \mathrm{~h}$ before and throughout the infection.

Bacterial strains. Three prototype $C$. jejuni strains were used, to represent a variety of potential virulence mechanisms. NCTC11168, the first sequenced C. jejuni strain (1); the invasive strain, ATCC81-176 (3); TGH9011 (ATCC43431), a clinical isolate characterized at the University of Toronto, and a noninvasive $f g F$ isogenic mutant of TGH9011 (17). All strains were incubated in Mueller-Hinton broth and regrown on Columbia-blood-agar plates under microaerophilic conditions $\left(48 \mathrm{~h} ; 37^{\circ} \mathrm{C}\right)$.

Enterohemorrhagic Escherichia coli (EHEC), strain CL56 (serotype O157:H7) (18) was grown in Penassay-broth overnight, and regrown in 10:1 fresh Penassay-broth $\left(3 \mathrm{~h} ; 37^{\circ} \mathrm{C}\right)$. Multiplicity of infection (MOI) used for all experiments was 100:1, except for invasion assays (20:1).

Bacterial invasion and translocation. For invasion assays, epithelial cells were infected with $C$. jejuni strains. After various time points (1-48 h), wells were washed three times with PBS to remove nonadherent bacteria; fresh antibiotic-free cell culture medium was then added $\left(2 \mathrm{~h} ; 37^{\circ} \mathrm{C}\right)$ with or without gentamicin (Sandoz, Boucherville, Quebec, Canada). Gentamicin $(100 \mu \mathrm{g} / \mathrm{mL})$ effectively killed all bacteria (11). Cells were then washed and lysed with $0.1 \%$ Triton X-100 (15 min; $20^{\circ} \mathrm{C}$; Sigma Chemical Co.). Aliquots $(10 \mu \mathrm{L})$ were plated onto blood-agar plates in serial 10-fold dilutions and grown under microaerophilic conditions $\left(48 \mathrm{~h} ; 37^{\circ} \mathrm{C}\right)$. Bacteria recovered from wells with gentamicin represent only internalized organisms, whereas those present in wells without antibiotics represent both adherent and invasive bacteria. Invasion, as percentage of bacterial adhesion, which is an expression of the ability of adherent bacteria to invade cells (19), was calculated by a ratio of enumerated colony-forming units in each well. Bacterial translocation was measured by sampling $10 \mu \mathrm{L}$ from the basal compartment of $3 \mu$-pore Transwells for determination of colony-forming units.

Measurement of transepithelial electrical resistance (TER) and macromolecular permeability. MDCK-I and T84 cells were plated onto Transwells $\left(5 \times 10^{4}\right.$ or $2 \times 10^{5}$ cells/well, respectively; $6.5 \mathrm{~mm}$ diameter; $0.4 \mu$-pore size; Corning) and grown until apical junctional complexes developed. Transwells were infected apically with either EHEC O157:H7 or $C$. jejuni strains (MOI: $100: 1 ; 37^{\circ} \mathrm{C} ; 5 \% \mathrm{CO}_{2}$ ). TER was measured prior to, $24 \mathrm{~h}$, and $48 \mathrm{~h}$ after infection using a Millicell-ERS Voltmeter and chopstick electrodes (Millpore, Bedford, MA). TER of Transwells without cells was $32 \Omega \cdot \mathrm{cm}^{2}$.

Dextran flux was used to measure paracellular macromolecular permeability (20). After $48 \mathrm{~h}$ of infection, monolayers were washed four times with PBS, and infrared-labeled dextran $(10 \mathrm{kD} ; 0.2 \mathrm{~mL}$ of $0.1 \mathrm{mg} / \mathrm{mL}$ in DMEM; Alexa-Fluor 647, Molecular Probes, Eugene, OR) was then inserted into the apical compartment of Transwells. After $5 \mathrm{~h}$ at $37^{\circ} \mathrm{C}$, the basal compartment was sampled, diluted 1:20, and loaded into 96-well plates for infrared signal quantification using an imaging system at $700 \mathrm{~nm}$ (Odyssey, Licor, Rockford, IL). Integrated intensities were expressed relative to uninfected polarized monolayers.

Transmission electron microscopy. Confluent T84 Transwells were infected with $C$. jejuni, strain 81-176 (MOI: 100:1; 48 h; $37^{\circ} \mathrm{C}$ ). Support membranes were washed, excised, and cells fixed in formaldehyde (4\%) and glutaraldehyde $(1 \%)$ in phosphate buffer, and postfixed in osmium tetroxide $\left(1 \% ; 2 \mathrm{~h} ; 20^{\circ} \mathrm{C}\right)$. Specimens were then dehydrated, embedded, and stained with uranyl acetate and lead citrate, and examined by transmission electron microscopy (JEM-1011, JEOL USA Corp., Peabody, MA) at 75 kV. Desmosome width was assessed by measuring spaces between adjacent membranes of well-oriented desmosomes.

Statistics. Results are expressed as means \pm SEM. $N$ represents the number of individual experiments. ANOVA and unpaired $t$ test were conducted using InStat3 (GraphPad, San Diego, CA).
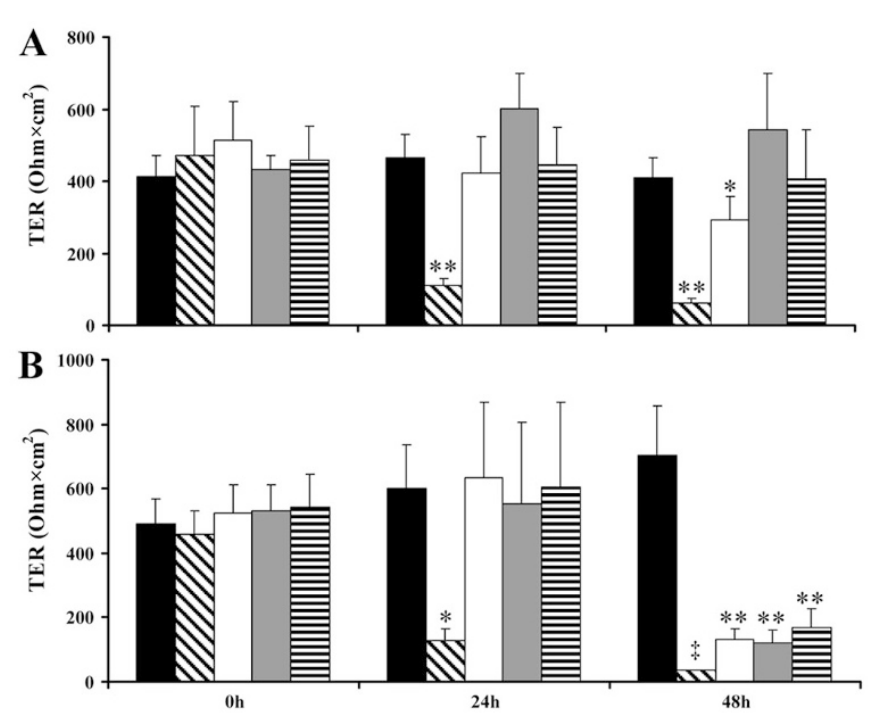

Figure 1. C. jejuni decreases TER of epithelial cell monolayers after $48 \mathrm{~h}$ of infection. Epithelial cells were infected with EHEC 0157:H7 or C. jejuni

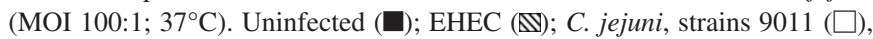
11168 ( $\square$ ), and 81-176 (司). Panel A, Only C. jejuni, strain 9011 significantly reduced TER by $24.7 \pm 8.3 \%$ after $48 \mathrm{~h}$ in MDCK-I cells. Panel $B$, Infection of T84 cell monolayers with all $C$. jejuni strains caused a comparable decrease in TER, after $48 \mathrm{~h}(77.3 \pm 8.3 \%, 78.0 \pm 8.8 \%$, and $72.8 \pm 9.1 \%$ for strains 9011,11168 , and $81-176$, relative to uninfected cells, respectively), but not after $24 \mathrm{~h}$. EHEC O157:H7 caused a significant reduction in TER in MDCK-I $($ Panel $A)$ and T84 (Panel $B)$ cells at all time points tested. Mean \pm SEM; $n=$ 4-6; ANOVA: $* p<0.05, * * p<0.01, \neq p<0.001$.

\section{RESULTS}

C. jejuni decreases TER of T84 monolayers after $48 \mathrm{~h}$. In contrast to the rapid reduction in TER with EHEC O157:H7 infection, no reduction was demonstrated after $24 \mathrm{~h}$ of $C$. jejuni infection in both MDCK-I and T84 cells (Fig. $1 A$ and $B)$. However, a $25 \%$ reduction in TER was observed after $48 \mathrm{~h}$ infection with $C$. jejuni, strain 9011 in MDCK-I cells (Fig. $1 A)$. In contrast to canine kidney-derived MDCK-I monolayers, which were less susceptible to $C$. jejuni-mediated barrier disruption, all three tested $C$. jejuni strains caused a comparable reduction of $73-78 \%$ in TER in human intestinal T84 cells after $48 \mathrm{~h}$ (Fig. $1 B$ ). Infection with a noninvasive $C$. jejuni $f g F$ deficient mutant did not reduce TER $(n=2)$. Live bacteria were required, because there was no drop in TER with heat-inactivated $(n=4)$ or formaldehyde-fixed bacteria $(n=$ 2 ). The effects were not due to the metabolic activity of bacteria on epithelial cells, because incubation with tissue culture medium corrected to $\mathrm{pH} 6$ (the $\mathrm{pH}$ of medium after $48 \mathrm{~h}$ of infection; $n=2$ ) and conditioned medium did not reduce TER $(n=2)$.

C. jejuni infection increases macromolecular permeability of T84 monolayers. Transcytosis of $10 \mathrm{kD}$ dextran across monolayers supported the TER results. Consistent with previous reports (21), EHEC O157:H7 caused a dramatic increase in permeability to dextran in both epithelial cell types, indicating breakdown of the epithelial barrier. Infection with $C$. jejuni resulted in increased dextran permeability in T84 cells, but to a lesser degree relative to EHEC infection (Fig. 2).

Intercellular spaces of T84 monolayers are modified following C. jejuni infection. Transmission electron microscopy 
A

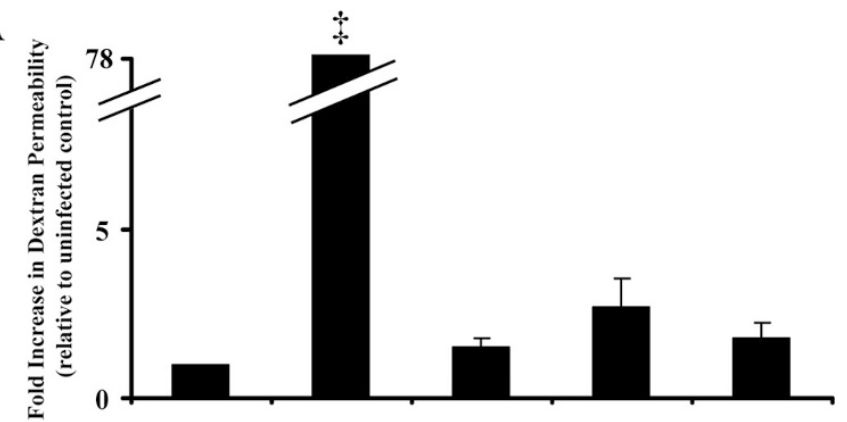

B

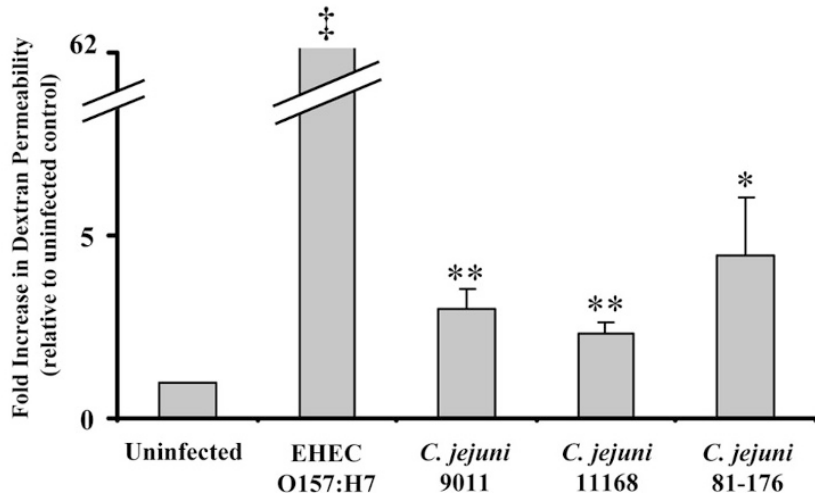

Figure 2. C. jejuni infection increases T84 cell monolayer permeability. After $48 \mathrm{~h}$ of infection of monolayers grown on Transwells, an infraredlabeled dextran $(10 \mathrm{kD})$ probe was placed into the apical compartment and sampled $5 \mathrm{~h}$ later from the basal compartment. Samples were then diluted and the infrared signal was quantified. EHEC infection resulted in a 78- and 62-fold increase in permeability to dextran in MDCK-I (Panel A) and T84 (Panel B) cells, respectively. C. jejuni infection caused a 2.3-4.5-fold increase in permeability to dextran, relative to uninfected controls, respectively, but only in T84 cell monolayers (Panel B). Average dextran concentration in the basal compartment of uninfected wells was $8 \pm 2$ and $18 \pm 3 \mathrm{ng} / \mathrm{mL}$ in MDCK-I and T84 Transwells, respectively. Mean \pm SEM; $n=4-5$; ANOVA: $\neq p<0.001 ; t$ test: $* p<0.05, * * p<0.01$.

of infected T84 monolayers supported the findings observed by TER and dextran permeability. Relative to uninfected cells (Fig. 3A-E), C. jejuni infection disrupted intercellular spaces with the formation of larger gaps between adjacent T84 cells at $48 \mathrm{~h}$ (Fig. $3 F-K$ ), but not after $18 \mathrm{~h}$ infection (data not shown). Although the morphology of tight junctions remained unchanged (Fig. $3 F-I$ ), the intercellular portion of desmosomes was increased following infection, when compared with uninfected T84 monolayers (Fig. $3 I-K$ and $3 D-E$, respectively; quantified in Fig. $3 N$ ). Invasive bacteria were detected in the cytosol of infected cells (Fig. $3 L$ and $M$ ).

T84 cells are susceptible to $\mathrm{C}$. jejuni invasion. To identify mechanisms responsible for the observed sensitivity of T84 monolayers to Campylobacter-induced barrier damage, the ability of $C$. jejuni to invade four epithelial cell lines was assessed. C. jejuni, strain 9011 was more invasive in T84 cells than in three other epithelial cell lines (Fig. 4), including both polarized (MDCK-I) and nonpolarized (HEp-2 and Intestine 407) cells, all previously used to assess microbial invasion (22,23). C. jejuni, strain 81-176 also showed increased invasion of T84 cells, whereas C. jejuni, strain 11168 demonstrated a trend for increased invasion of $\mathrm{T} 84$, relative to MDCK-I cells.

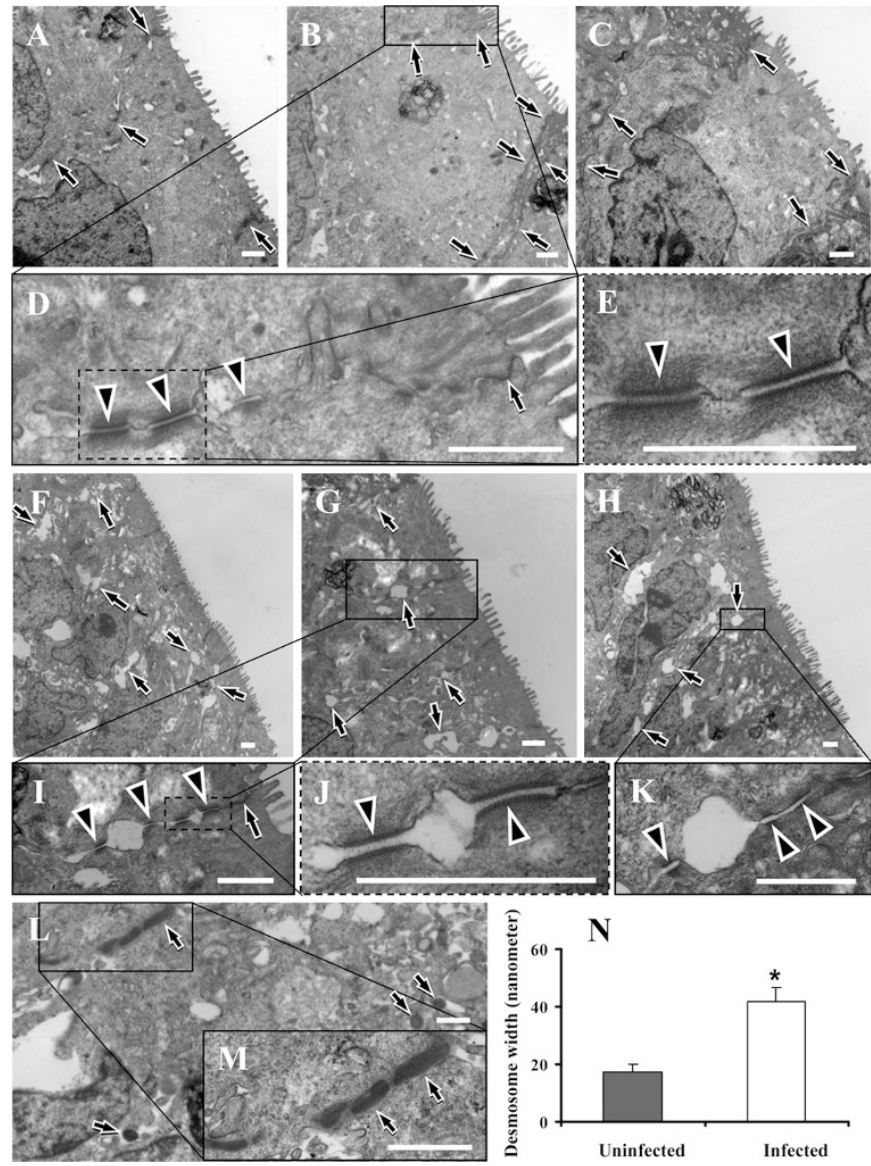

Figure 3. Bacterial disruption of intercellular junctional complexes. Confluent T84 monolayers were either left uninfected or infected with $C$. jejuni, strain 81-176 (MOI: 100:1; 48 h). Panels $A-C$, Uninfected T84 monolayers with normal intercellular junctions (arrows). Panel $D$, Higher magnification of apical junctional complexes of uninfected T84 monolayers [Panel E, Enlarged insert demonstrating normal desmosomes (arrowheads)]. Panels $F-H$, T84 monolayers after $48 \mathrm{~h}$ infection with $C$. jejuni, strain 81-176 showing multiple gaps between cells (arrows) with intact tight junctions. Panels I, $J$ (insert of Panel $I$ ) and $K$ demonstrate abnormal desmosomes with widened gaps in infected T84 monolayers (arrowheads), but normal tight junctions (arrow in Panel I). Panels $L$ and $M$, invasive bacteria in infected T84 cells (arrows). Panel N, Desmosomes of infected T84 monolayers had double the width of uninfected monolayers. Thirteen and eleven desmosomes were measured for uninfected and infected monolayers, respectively (mean \pm SEM; $t$ test: $* p<0.0001)$. Measurement bar: $1 \mu$.

Epithelial cell invasion and bacterial translocation precede increased monolayer permeability. To determine a temporal connection between $C$. jejuni internalization and monolayer permeability, bacterial invasion, translocation across monolayers, and TER were assessed over $48 \mathrm{~h}$ in the same Transwells. Invasion and translocation of $C$. jejuni, strain 81-176 into T84 cells were apparent as of 1 and $3 \mathrm{~h}$ after infection, respectively, while TER remained at control levels until $48 \mathrm{~h}$ postinfection (Fig. 1). Taken together, these results indicate that $C$. jejuni first invades epithelial cells and then, similar to other enteropathogens (18), transcytoses across the monolayer without compromising intercellular junctions that are disrupted later during the infectious process.

C. jejuni were introduced directly into the basolateral compartment of Transwells ( $3 \mu$-pore size), to bypass the need for bacterial transcytosis via cellular invasion and translocation. 


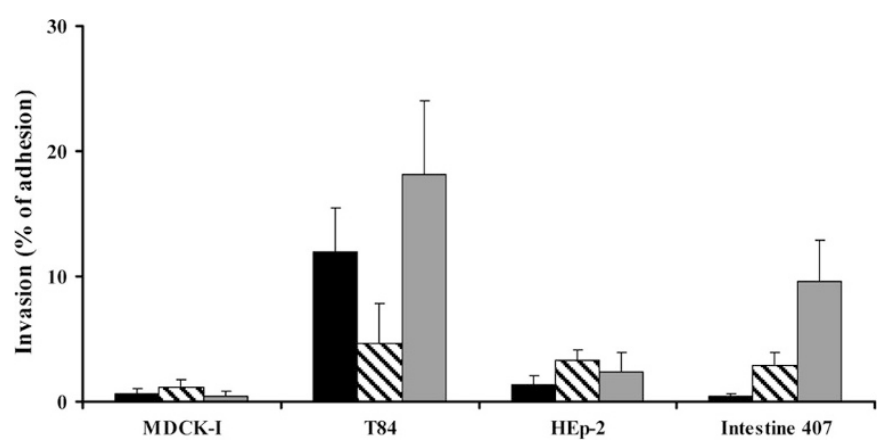

Figure 4. C. jejuni invasion into epithelial cells is strain- and cell typespecific. MDCK-I, T84, HEp-2, and Intestine 407 cells were grown to confluence in 24-mm wells and infected with prototype $C$. jejuni strains (MOI: $\left.20: 1 ; 4 \mathrm{~h} ; 37^{\circ} \mathrm{C}\right)$ and then incubated with or without gentamicin (100 $\mu \mathrm{g} / \mathrm{mL} ; 2 \mathrm{~h}$ ). Epithelial cells were then lysed in $0.1 \%$ Triton-X and plated in serial 10-fold dilutions. Invasion is presented as the percentage of bacterial adhesion. C. jejuni, strain 9011 (ם) showed higher invasion efficiency into T84 cells $(12 \pm 3.5 \%)$ relative to the three other cell-lines (HEp-2: $1.4 \pm$ 0.7\%; MDCK-I: $0.6 \pm 0.4 \%$; Intestine 407: $0.4 \pm 0.2 \% ; n=4-5$; ANOVA:

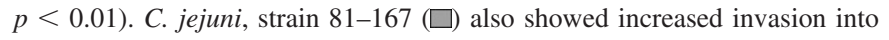
T84 cells $(18.1 \pm 5.9 \%$ vs. $0.4 \pm 0.3 \%$, in MDCK-I cells; $n=4-7 ; p<$ $0.05)$, and a trend for increased invasion of strain $11168(\mathbb{\nabla})$ into T84 cells was demonstrated $(4.7 \pm 3.2 \%$ vs. $1.1 \pm 0.6 \%$ in MDCK-I cells; $n=4-5$; $p=0.07)$. In Intestine 407 cells $C$. jejuni, strain 81-176 was more invasive than strain $9011(n=4-5 ; t$ test: $p=0.03)$.

However, this approach did not enhance the drop in permeability (data not shown) because basolateral infection only shortened the process by the $3 \mathrm{~h}$ required for bacteria to translocate the monolayer, whereas TER is reduced later in the infectious process. Colchicine $(5-50 \mu \mathrm{g} / \mathrm{mL})$, reported to inhibit bacterial invasion in intestine 407 cells (8), did not reduce $C$. jejuni invasion of T84 cells (data not shown) and, therefore, was not useful for evaluating the association between invasion and permeability.

PI3-K inhibition reduces bacterial invasion and blocks the drop in TER in C. jejuni-infected T84 monolayers. Consistent with previous reports (24), there was reduced invasion of C. jejuni, strain 81-176 into T84 cells after pretreatment with the pharmacological inhibitor of PI3-K, LY294002. A higher concentration of the PI3-K inhibitor $(100 \mu \mathrm{M})$ resulted in a larger reduction in bacterial invasion, whereas pretreatment with the inactive analog had no effect on microbial invasion (Fig. 5A). LY294002 pretreatment of polarized monolayers also attenuated the $C$. jejuni-induced drop in TER, thereby providing evidence that the effects of $C$. jejuni on epithelial barrier dysfunction were mediated by bacterial invasion. By contrast, pretreatment with the inactive analog had no effect on C. jejuni-induced drop in TER (Fig. 5B). Furthermore, the protective effect of LY294002 on C. jejuni-mediated barrier disruption may be underestimated, because LY294002 itself attenuated the increase in TER to $65.6 \pm 16.6 \%$ of uninfected controls. The inability of the PI3-K inhibitor to completely protect against $C$. jejuni-mediated barrier disruption may be attributed to partial inhibition of bacterial invasion (Fig. 5A). Taken together, the findings indicate that $C$. jejuni-induced disruption of polarized monolayers is mediated by invasion of organisms into the cytoplasm of infected epithelial cells.

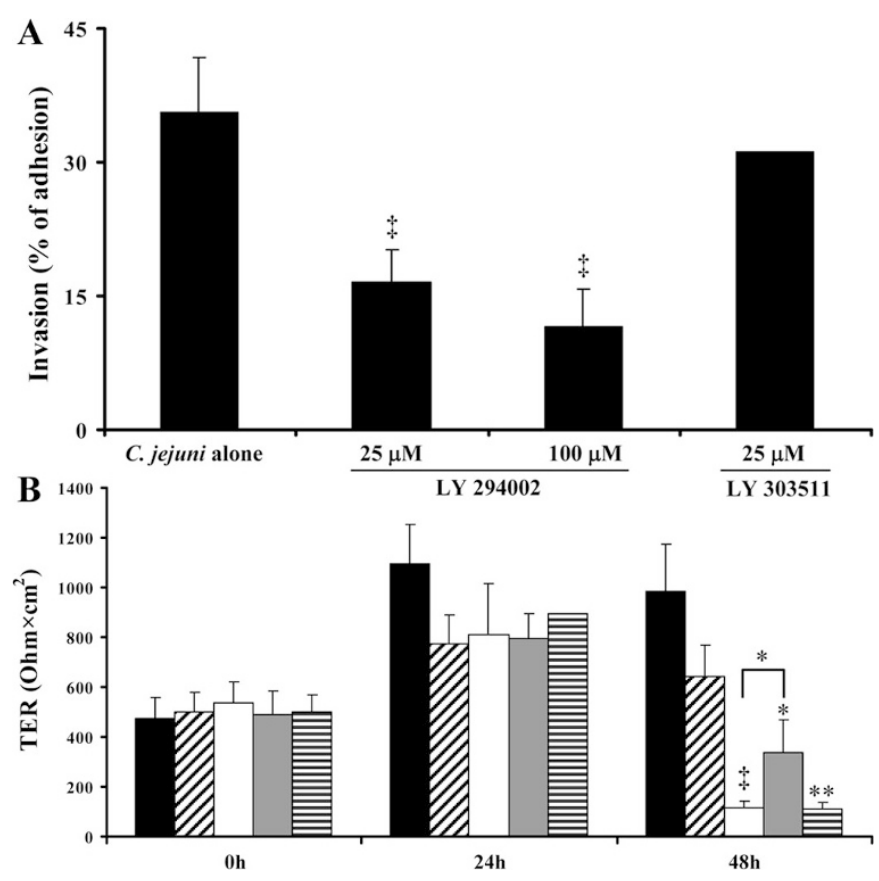

Figure 5. PI3-K inhibition decreases bacterial invasion and reduces $C$. jejuni-induced barrier disruption in T84 monolayers. T84 cells were pretreated with either the PI3-K inhibitor LY294002 or an inactive analog, LY303511 $\left(1 \mathrm{~h} ; 37^{\circ} \mathrm{C}\right)$ before infection with C. jejuni, strain 81-176. Panel A, Invasion into T84 epithelial cells, measured $4 \mathrm{~h}$ after infection, was inhibited by $53 \pm 7.5 \%$ and $72 \pm 3 \%$ with LY294002 pretreatment $(25$ and $100 \mu \mathrm{M}$, respectively), but not with the inactive analog $(25 \mu \mathrm{M})$. Panel B, C. jejuni, strain 81-176, $48 \mathrm{~h}$ infection of T84 monolayers reduced TER without the PI3-K inhibitor $(\square)$ or with the inactive analog (目) $(11.3 \pm 1.6 \%$ and $12.7 \pm$ $2.4 \%$ of control, respectively), but to just $34.8 \pm 10.9 \%$ of control values with the active inhibitor ( $\square$ ). Uninfected ( $\square$ ); LY249002 alone (响).

\section{DISCUSSION}

This study shows that $C$. jejuni infection of polarized epithelial monolayers induces cell-type specific alterations in polarized epithelial monolayers. The findings in the present study show that, similar to other intestinal pathogens $(18,21)$, C. jejuni infection inflicts changes in epithelial barrier function, thereby leading to potential exposure of the subepithelial immune components to chronic antigenic stimulation. Such changes in epithelial permeability are well documented in humans with IBD (13), to which $C$. jejuni infection has been linked $(2,5,6)$.

Other studies have shown increased permeability of human polarized intestinal cells (T84 and Caco-2) infected by $C$. jejuni (25-28). Similar to our findings, Chen et al. (25) observed a delayed effect of $C$. jejuni on T84 cells resulting in activation of innate immunity. In contrast, other studies using Caco-2 cells, which attain lower baseline TER, report a more variable drop in resistance $(26,27)$. Such cell type-dependent variability prompted us to study the effects of $C$. jejuni infection in epithelial cells with different characteristics. We, therefore, used transformed human colonic T84 cells and canine kidney MDCK-I cells as model polarized epithelium, which both express mature apical junctional complex proteins and maintain cell polarity (29), and are used extensively to study host-microbial interactions $(15,30)$. 
Lack of morphologic alterations of tight junctions of cells infected with $C$. jejuni is in keeping with previous observations regarding ZO-1 (25). Changes in desmosomes of infected T84 cells, observed by transmission electron microscopy, have not been previously described, and contrast the lack of effect of pathogenic E. coli and Citrobacter rodentium on desmosomes (31). Since desmosomes are involved in cell-cell contact and in anchoring the cytoskeleton, separation of desmosomes could contribute to epithelial barrier dysfunction. However, it remains unclear whether alterations in desmosomes observed in the present study are the reason for increased permeability (32). Further studies, focusing on the effects of bacterial infection on proteins involved in maintenance of desmosomes, such as desmoglein and desmoplakin, may provide additional insight.

Our findings clearly demonstrate increased vulnerability of human intestinal (T84) cells following $C$. jejuni challenge, relative to renal tubule-derived canine (MDCK-I) cells. The increased susceptibility of T84 cells was demonstrated by TER reduction and was also reflected by an increase in macromolecular permeability, relative to MDCK-I monolayers. There are several potential explanations for the differences observed. T84 cells produce large amounts of mucin, which act as a chemoattractant for $C$. jejuni (33). However, there was no increase in bacterial adherence to T84 cells (data not shown). Therefore, binding of $C$. jejuni to mucins is not likely to be responsible for the greater susceptibility of T84 cells to changes in barrier function. Another difference between the two polarized epithelial cell lines is in the expression of tight junction proteins. MDCK-I cells achieve high resistance, at least in part, due to the absence of claudin-2, which results in more 'leaky' junctions (34), whereas T84 cells express claudin-2 (35). Such differences could contribute to varying susceptibility to $C$. jejuni-induced barrier disruption. Interestingly, patients with IBD demonstrate a relative increase in the expression of claudin-2 and a decrease in other claudins, which correlates with increased gut permeability (36).

In addition to effects on permeability, the $C$. jejuni strains tested were also more effectively internalized into T84 cells than MDCK-I cells. Because the susceptibility of T84 cells to both barrier disruption and invasion by the three strains was comparable, the observed effects could represent a mechanism that is common to all $C$. jejuni strains. It is possible that bacterial invasion into polarized epithelial cells is dependent on the modification of junctional physiology and paracellular passage of the organism (37). However, if this were the case, a drop in TER might have been expected before bacterial invasion and translocation. In contrast, our findings indicate an opposite sequence of events, where bacteria enter into infected eukaryotic cells to induce changes in intercellular junctions by altering signal transduction pathways. A similar sequence of events has also been described for other invasive pathogens (38), but contrasts with Helicobacter pylori infection, which is reported to alter the physiology of apical junctional complexes without entering into the cytoplasm (39).

To determine whether effects on barrier function were dependent on bacterial internalization into the cytosol, invasion of C. jejuni was inhibited using a pharmacological inhib- itor of PI3-K. As previously reported (24), this inhibitor reduced invasion of $C$. jejuni. The same inhibitor also prevents interferon- $\gamma$-induced barrier disruption, but not disruption by enteropathogenic E. coli (40), which argues against direct protection from barrier disruption induced by bacteria. The partial reduction in barrier disruption with inhibition of PI3-K suggests the involvement of additional mechanisms, such as displacement of occludin from lipid-rafts (25). Recent findings indicate that bacterial invasion is also reduced using inhibitors of Rho GTPases (41), which are involved in regulation of intercellular junctions (42).

Taken together, the results of the present study indicate that epithelial barrier disruption induced by $C$. jejuni infection depends on bacterial invasion. An improved understanding of the precise mechanisms leading to $C$. jejuni-induced diarrhea, intestinal injury, and chronic immune stimulation is essential for developing strategies to interrupt the infectious process and prevent its complications.

\section{REFERENCES}

1. Dorrell N, Wren BW 2007 The second century of Campylobacter research: recent advances, new opportunities and old problems. Curr Opin Infect Dis 20:514-518

2. Ternhag A, Torner A, Svensson A, Ekdahl K, Giesecke J 2008 Short- and long-term effects of bacterial gastrointestinal infections. Emerg Infect Dis 14:143-148

3. Crushell E, Harty S, Sharif F, Bourke B 2004 Enteric Campylobacter: purging its secrets? Pediatr Res 55:3-12

4. Marshall JK, Thabane M, Garg AX, Clark WF, Salvadori M, Collins SM 2006 Incidence and epidemiology of irritable bowel syndrome after a large waterborne outbreak of bacterial dysentery. Gastroenterology 131:445-450

5. Garcia Rodriguez LA, Ruigomez A, Panes J 2006 Acute gastroenteritis is followed by an increased risk of inflammatory bowel disease. Gastroenterology 130:15881594

6. Boyanova L, Gergova G, Spassova Z, Koumanova R, Yaneva P, Mitov I, Derejian S, Krastev Z 2004 Campylobacter infection in 682 Bulgarian patients with acute enterocolitis, inflammatory bowel disease, and other chronic intestinal diseases. Diagn Microbiol Infect Dis 49:71-74

7. Young KT, Davis LM, DiRita VJ 2007 Campylobacter jejuni: molecular biology and pathogenesis. Nat Rev Microbiol 5:665-679

8. Biswas D, Itoh K, Sasakawa C 2003 Role of microfilaments and microtubules in the invasion of INT-407 cells by Campylobacter jejuni. Microbiol Immunol 47:469473

9. Fox JG, Rogers AB, Whary MT, Ge Z, Taylor NS, Xu S, Horwitz BH, Erdman SE 2004 Gastroenteritis in NF-kappaB-deficient mice is produced with wild-type Campylobacter jejuni but not with $C$. jejuni lacking cytolethal distending toxin despite persistent colonization with both strains. Infect Immun 72:1116-1125

10. Bacon DJ, Alm RA, Burr DH, Hu L, Kopecko DJ, Ewing CP, Trust TJ, Guerry P 2000 Involvement of a plasmid in virulence of Campylobacter jejuni 81-176. Infect Immun 68:4384-4390

11. Watson RO, Galan JE 2008 Campylobacter jejuni survives within epithelial cells by avoiding delivery to lysosomes. PLoS Pathog 4:e14

12. Poly F, Read T, Tribble DR, Baqar S, Lorenzo M, Guerry P 2007 Genome sequence of a clinical isolate of Campylobacter jejuni from Thailand. Infect Immun 75:34253433

13. Xavier RJ, Podolsky DK 2007 Unravelling the pathogenesis of inflammatory bowel disease. Nature 448:427-434

14. Sartor RB 2008 Microbial influences in inflammatory bowel diseases. Gastroenterology 134:577-594

15. Amieva MR, Vogelmann R, Covacci A, Tompkins LS, Nelson WJ, Falkow S 2003 Disruption of the epithelial apical-junctional complex by Helicobacter pylori CagA. Science 300:1430-1434

16. Johnson-Henry KC, Donato KA, Shen-Tu G, Gordanpour M, Sherman PM 2008 Lactobacillus rhamnosus strain GG prevents enterohemorrhagic Escherichia coli O157:H7-induced changes in epithelial barrier function. Infect Immun 76:13401348

17. Song YC, Jin S, Louie H, Ng D, Lau R, Zhang Y, Weerasekera R, Al Rashid S, Ward LA, Der SD, Chan VL 2004 FlaC, a protein of Campylobacter jejuni TGH9011 (ATCC43431) secreted through the flagellar apparatus, binds epithelial cells and influences cell invasion. Mol Microbiol 53:541-553

18. Zareie M, Riff J, Donato K, McKay DM, Perdue MH, Soderholm JD, Karmali M, Cohen MB, Hawkins J, Sherman PM 2005 Novel effects of the prototype translocating Escherichia coli, strain C25 on intestinal epithelial structure and barrier function. Cell Microbiol 7:1782-1797

19. Jones N, Perdue MH, Sherman PM, McKay DM 2002 Bacterial interactions with host epithelium in vitro. Methods Mol Biol 188:383-400

20. Raimondi F, Santoro P, Barone MV, Pappacoda S, Barretta ML, Nanayakkara M, Apicella C, Capasso L, Paludetto R 2008 Bile acids modulate tight junction structure 
and barrier function of Caco-2 monolayers via EGFR activation. Am J Physiol Gastrointest Liver Physiol 294:G906-G913

21. Howe KL, Reardon C, Wang A, Nazli A, McKay DM 2005 Transforming growth factor-beta regulation of epithelial tight junction proteins enhances barrier function and blocks enterohemorrhagic Escherichia coli O157:H7-induced increased permeability. Am J Pathol 167:1587-1597

22. Meraz IM, Arikawa K, Ogasawara J, Hase A, Nishikawa Y 2006 Epithelial cells secrete interleukin- 8 in response to adhesion and invasion of diffusely adhering Escherichia coli lacking Afa/Dr genes. Microbiol Immunol 50:159-169

23. Kierbel A, Gassama-Diagne A, Mostov K, Engel JN 2005 The phosphoinositol-3kinase-protein kinase B/Akt pathway is critical for Pseudomonas aeruginosa strain PAK internalization. Mol Biol Cell 16:2577-2585

24. Hu L, McDaniel JP, Kopecko DJ 2006 Signal transduction events involved in human epithelial cell invasion by Campylobacter jejuni 81-176. Microb Pathog 40:91-100

25. Chen ML, Ge Z, Fox JG, Schauer DB 2006 Disruption of tight junctions and induction of proinflammatory cytokine responses in colonic epithelial cells by Campylobacter jejuni. Infect Immun 74:6581-6589

26. Cogan TA, Thomas AO, Rees LE, Taylor AH, Jepson MA, Williams PH, Ketley J, Humphrey TJ 2007 Norepinephrine increases the pathogenic potential of Campylobacter jejuni. Gut 56:1060-1065

27. MacCallum A, Hardy SP, Everest PH 2005 Campylobacter jejuni inhibits the absorptive transport functions of Caco-2 cells and disrupts cellular tight junctions. Microbiology 151:2451-2458

28. Bras AM, Ketley JM 1999 Transcellular translocation of Campylobacter jejuni across human polarised epithelial monolayers. FEMS Microbiol Lett 179:209-215

29. Ivanov AI, Hunt D, Utech M, Nusrat A, Parkos CA 2005 Differential roles for actin polymerization and a myosin II motor in assembly of the epithelial apical junctional complex. Mol Biol Cell 16:2636-2650

30. Bagnoli F, Buti L, Tompkins L, Covacci A, Amieva MR 2005 Helicobacter pylori CagA induces a transition from polarized to invasive phenotypes in MDCK cells. Proc Natl Acad Sci U S A 102:16339-16344

31. Guttman JA, Kazemi P, Lin AE, Vogl AW, Finlay BB 2007 Desmosomes are unaltered during infections by attaching and effacing pathogens. Anat Rec (Hoboken) 290:199-205
32. Amagai M, Matsuyoshi N, Wang ZH, Andl C, Stanley JR 2000 Toxin in bullous impetigo and staphylococcal scalded-skin syndrome targets desmoglein 1. Nat Med 6:1275-1277

33. McAuley JL, Linden SK, Png CW, King RM, Pennington HL, Gendler SJ, Florin TH, Hill GR, Korolik V, McGuckin MA 2007 MUC1 cell surface mucin is a critical element of the mucosal barrier to infection. J Clin Invest 117:2313-2324

34. Furuse M, Furuse K, Sasaki H, Tsukita S 2001 Conversion of zonulae occludentes from tight to leaky strand type by introducing claudin-2 into Madin-Darby canine kidney I cells. J Cell Biol 153:263-272

35. Prasad S, Mingrino R, Kaukinen K, Hayes KL, Powell RM, MacDonald TT, Collins JE 2005 Inflammatory processes have differential effects on claudins 2, 3 and 4 in colonic epithelial cells. Lab Invest 85:1139-1162

36. Zeissig S, Burgel N, Gunzel D, Richter J, Mankertz J, Wahnschaffe U, Kroesen AJ, Zeitz M, Fromm M, Schulzke JD 2007 Changes in expression and distribution of claudin 2, 5 and 8 lead to discontinuous tight junctions and barrier dysfunction in active Crohn's disease. Gut 56:61-72

37. Konkel ME, Christensen JE, Keech AM, Monteville MR, Klena JD, Garvis SG 2005 Identification of a fibronectin-binding domain within the Campylobacter jejuni $\mathrm{CadF}$ protein. Mol Microbiol 57:1022-1035

38. Hanajima-Ozawa M, Matsuzawa T, Fukui A, Kamitani S, Ohnishi H, Abe A, Horiguchi Y, Miyake M 2007 Enteropathogenic Escherichia coli, Shigella flexneri, and Listeria monocytogenes recruit a junctional protein, zonula occludens-1, to actin tails and pedestals. Infect Immun 75:565-573

39. Saadat I, Higashi H, Obuse C, Umeda M, Murata-Kamiya N, Saito Y, Lu H, Ohnishi N, Azuma T, Suzuki A, Ohno S, Hatakeyama M 2007 Helicobacter pylori CagA targets PAR1/MARK kinase to disrupt epithelial cell polarity. Nature 447:330-333

40. McKay DM, Watson JL, Wang A, Caldwell J, Prescott D, Ceponis PM, Di Leo V, Lu J 2007 Phosphatidylinositol 3'-kinase is a critical mediator of interferon-gammainduced increases in enteric epithelial permeability. J Pharmacol Exp Ther 320:1013-1022

41. Krause-Gruszczynska M, Rohde M, Hartig R, Genth H, Schmidt G, Keo T, Konig W, Miller WG, Konkel ME, Backert S 2007 Role of the small Rho GTPases Rac1 and Cdc42 in host cell invasion of Campylobacter jejuni. Cell Microbiol 9:24312444

42. Otani T, Ichii T, Aono S, Takeichi M 2006 Cdc42 GEF Tuba regulates the junctional configuration of simple epithelial cells. J Cell Biol 175:135-146 